\title{
Motion Compensation of Tendon-Sheath Driven Continuum Manipulator for Endoscopic Surgery
}

\author{
K. C. Lau ${ }^{1 \star}$, Esther Y. Y. Leung ${ }^{1}$, Carmen C. Y. Poon ${ }^{2}$, Philip W. Y. Chiü ${ }^{2}$, James Y. W. Lau ${ }^{2}$ and Y. Yam ${ }^{1}$ \\ ${ }^{1}$ Department of Mechanical and Automation Engineering, The Chinese University of Hong Kong \\ ${ }^{2}$ Department of Surgery, The Chinese University of Hong Kong
}

\begin{abstract}
Tendon-sheath actuation mechanism is widely used in surgical robot, especially in endoscopic surgery, due to its capable of providing remote force and action transmission through long and flexible channel. However, hysteresis, backlash, nonlinear friction are the drawbacks of this mechanism. Our surgical robot use continuum manipulator which is useful in endoscopic surgery, due to its flexible and simple structure. Unlike other literatures that focus on tendon-sheath compensation only, the continuum manipulator is also taken into application level analysis. A model based feedforward motion compensation for tendon-sheath driven continuum manipulator is presented. The model is validated by using optical tracking system to trace the distal end position. Experiment result shows that the proposed model reduces the position error less than $5 \%$.
\end{abstract}

\section{Introduction}

Tendon-sheath actuation mechanism consists of a helical coil as the outer sheath, whereas steel wire as the inner tendon for force and action transmission. The wire is usually coated, for example by PTFE, to reduce the contact resistance between tendon and inner surface of the sheath. Tendon-sheath is able to transmit force from one end to another end by fixing the two ends of the sheath and pulling the inner tendon, no matter how the configuration of the middle part changes. It has the superior in working in narrow space application, such as robot hand ([2], [3], [4]), medical device ([5], [6]), industrial tele-operation system ([7], [8]), surgical robot ([9], [10]), etc.

Endoscopic surgery, also known as non-invasive surgery, makes use of natural open of human to reach target organ, for instance, from month to stomach and from anus to colon. This surgical task highly demand in using tendon-sheath actuation mechanism since the channel between opening and target place is long and tortuous, such as esophagus and colon. Tendon-sheath can be deformed to fit the shape of the channel to avoid damaging the inner organ, but still can transmit force and action from outside to inside human body. This mechanism is widely applied in endoscope nowadays.

However, tendon-sheath actuation mechanism usually has hysteresis, backlash, nonlinear friction behaviors that affects the performance of the system. The distal end output does not follow the input. However, sensors such as position and force sensor are very difficult to be placed at the distal end because of size problem. The feedback

\footnotetext{
*Corresponding author: kclau@mae.cuhk.edu.hk
}

loop cannot be closed. Therefore, most of the approach is to design a feedforward controller to handle this control problem. Model based controller usually considers the following parameters regarding to the tendon-sheath actuation mechanism components: sheath length, friction coefficient, total bending radius, pretension and tendon elasticity. In this paper, these parameters will be used to design a feedforward controller of friction, backlash and elongation compensation in tendon-sheath mechanism.

\section{Related Work}

Kaneko et al. ([1], [2]) were the first group studying the transmission characteristics of tendon-sheath. They developed a robot hand with the transmission model by considering a segment of tendon-sheath. A simple but effective Coulomb friction model was used. Palli et al. ([3], [4]) developed a tension controller by Coulomb friction model. Samuel et al. ([5], [6]) integrated the experiment results analysed by three-way ANOVA and gap width effect to build the backlash compensator for cardiac catheter. They also introduced a smoothing term in the compensator in order to smooth the transition when altering the tendon direction. Phee et al. ([11]-[14]) developed a flexible endoscopic system with tendon elongation compensation. The above systems or devices used single-tendon-sheath configuration in the modelling and experiments. In real life, double-tendon-sheath actuation is more practical because both forward and backward action can be controlled.

Agrawal et al. ([15]-[17]) described the characteristics of double-tendon-sheath by a set of partial differential

This is an Open Access article distributed under the terms of the Creative Commons Attribution License 4.0, which permits unrestricted use, distribution, and reproduction in any medium, provided the original work is properly cited. 


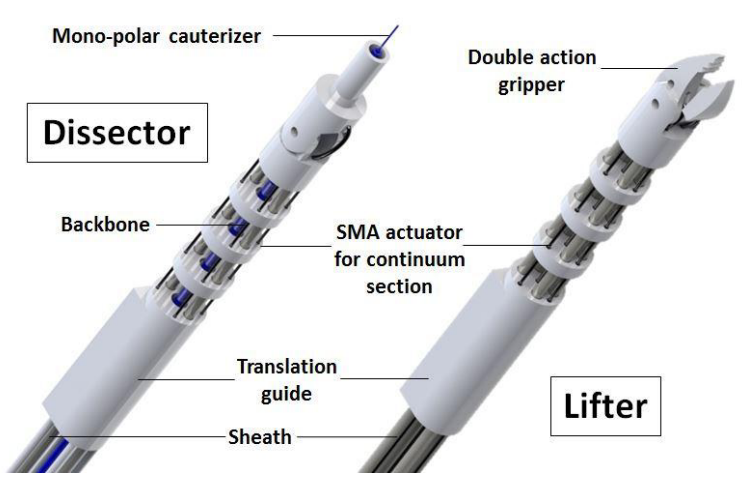

Fig. 1 Endoscopic surgical robot manipulator

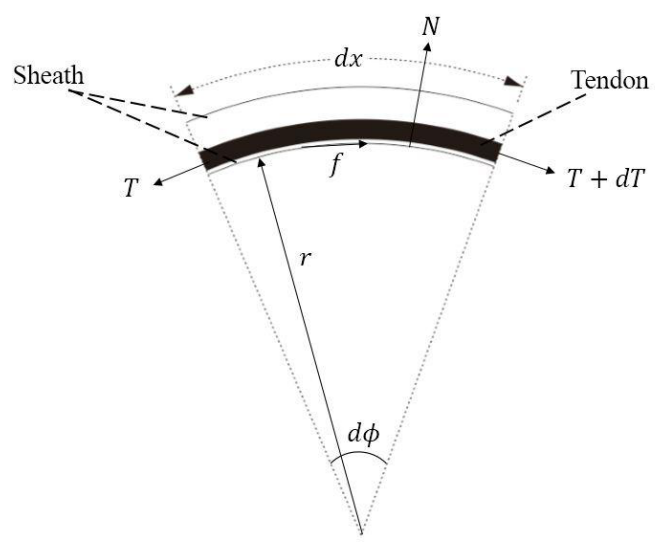

Fig. 2 Segment of tendon-sheath

equations (PDEs). S. C. Low, T. N. Do, Z. L. Sun ([18][20]) enhanced their endoscopic system by considering double-tendon-sheath into their modelling with the ability of distal end force estimation. Lin and Wang ([21]-[23]) developed a double tendon-sheath model by considering position and torque transmission characteristics.

In our previous studies ([24], [25]), we demonstrated our endoscopic robot was able to finish an endoscopic surgical task called Endoscopic Submucosal Dissection (ESD). The actuators of the robot consist of tendonsheath driven continuum manipulator with an addition end-effector, as shown in figure 1. We developed a compensation controller for single-tendon-sheath actuation mechanism ([26], [27]). The results showed that the compensation model was effective but not sufficient enough. Due to the novel design of our robot, doubletendon-sheath transmission and the actuation force of continuum manipulator have to be included into the model, which is different to other studies that only consider compensation of tendon-sheath transmission.

\section{System Modelling}

\subsection{Segment of Tendon-Sheath}

Figure 2 shows a segment of tendon-sheath. By balancing force on both ends, the force transmission model can be described by:

$$
\begin{aligned}
& d l=r d \phi \\
& N=T d \phi
\end{aligned}
$$

According to Coulomb friction model [28]:

$$
f=\mu \operatorname{sgn}(\dot{x})
$$

Introducing gap width effect [6]:

$$
f=\alpha \Delta D \mu N \operatorname{sgn}(\dot{x})
$$

Combining (1), (2) and (4) yields:

$$
\begin{aligned}
-d T & =\alpha \Delta D \mu T \frac{d x}{r} \operatorname{sgn}(\dot{x}) \\
T(x) & =T_{i n} e^{-\frac{\alpha \Delta D \mu}{r} x \operatorname{sgn}(\dot{x})} \\
& =T_{i n} e^{-\zeta \theta \operatorname{sgn}(\dot{x})}
\end{aligned}
$$

where

$T$ is the tension of tendon,

$f$ is the friction between tendon and sheath,

$N$ is the normal reaction force,

$r, d l, d \phi$ are the radius, arc length and bending angle of the tendon-sheath segment respectively,

$\mu$ is the friction constant,

$\Delta D$ is the gap width between tendon and sheath,

$\alpha$ is the parameter governing the gap width effect,

$\theta=\frac{x}{r}$ is the accumulated angle of the tendon-sheath,

$\zeta=\alpha \Delta D \mu$, and

$\operatorname{sgn}()$ is the sign function.

Suppose the configuration of the tendon-sheath does not change, the elongation $\delta(x)$ can be computed offline:

$$
\delta(x)=\frac{T(x)}{E}=\frac{1}{E} T_{i n} e^{-\zeta \theta \operatorname{sgn}(\dot{x})}
$$

The total elongation along the whole tendon-sheath:

$$
\begin{aligned}
\delta_{\text {total }}(x) & =\frac{1}{E} \int_{0}^{x} T_{\text {in }} e^{-\zeta \theta \operatorname{sgn}(\dot{x})} d x \\
& =\frac{T_{\text {in }} r}{E \zeta}\left(1-e^{-\zeta \theta \operatorname{sgn}(\dot{x})}\right) \\
& =\frac{r}{E \zeta}\left(T_{\text {in }}-T_{\text {in }} e^{-\zeta \theta \operatorname{sgn}(\dot{x})}\right) \\
& =\frac{r}{E \zeta}\left(T_{\text {in }}-T_{\text {out }}\right)
\end{aligned}
$$

As shown in (6), the tension of the tendon is exponentially decreased along the sheath. Once the input tension overcomes the friction along the whole sheath, there will be position change and tendon output at distal end. The output tension can be calculated according to (9), since the material properties, bending radius and input tension are known. 


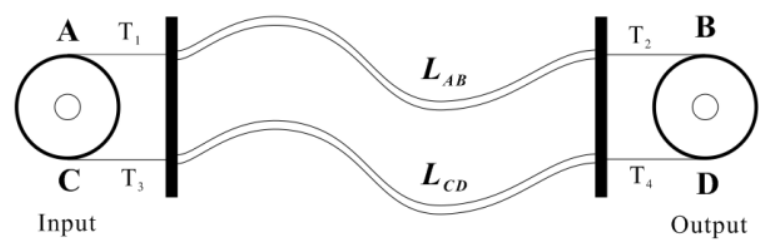

Fig. 3 Double tendon-sheath transmission system

\subsection{Double-Tendon-Sheath Transmission}

Figure 3 shows a double-tendon-sheath transmission system. Pulley on the left is the input and that on the right is output. Point $\mathrm{A}, \mathrm{B}, \mathrm{C}$ and $\mathrm{D}$ are the points that tendon just leave the pulley. The total length of tendon $A B$ is the displacement from $\mathrm{A}$ to $\mathrm{B}$ along the tendon, denoted by $L_{A B}$; total length of tendon $C D$ is the displacement from $\mathrm{C}$ to $\mathrm{D}$ along the tendon, denoted by $L_{C D}$. $T_{1}, T_{2}, T_{3}$ and $T_{4}$ are the tensions of the tendon at the location specified in the figure.

According to (6), assuming the input pulley rotates anti-clockwise, the tension at the output side can be expressed as:

$$
\begin{aligned}
& T_{2}=T_{1} e^{-\zeta \theta} \\
& T_{4}=T_{3} e^{\zeta \theta}
\end{aligned}
$$

Suppose $T_{0}$ is the pretension of both tendons and $L$ is the total length of the sheath. According to (8), the elongation of each tendon $\delta_{A B}$ and $\delta_{C D}$ can be calculated:

$$
\begin{aligned}
\delta_{A B} & =\frac{1}{E} \int_{0}^{L}\left(T_{1} e^{-\zeta \theta}-T_{0}\right) d x \\
& =\frac{1}{E}\left[\frac{T_{1} r}{\zeta}\left(1-e^{-\zeta \theta}\right)-T_{0} L\right] \\
\delta_{C D} & =\frac{1}{E} \int_{0}^{L}\left(T_{3} e^{\zeta \theta}-T_{0}\right) d x \\
& =-\frac{1}{E}\left[\frac{T_{3} r}{\zeta}\left(1-e^{\zeta \theta}\right)-T_{0} L\right]
\end{aligned}
$$

To shorten these expressions, let $v^{-}=\frac{r}{\zeta}\left(1-e^{-\zeta \theta}\right)$ and $v^{+}=\frac{r}{\zeta}\left(1-e^{\zeta \theta}\right)$, then:

$$
\begin{gathered}
\delta_{A B}=\frac{1}{E}\left[T_{1} v^{-}-T_{0} L\right] \\
\delta_{C D}=-\frac{1}{E}\left[T_{3} v^{+}-T_{0} L\right]
\end{gathered}
$$

Noted that $\delta_{A B}$ is positive and $\delta_{C D}$ is negative. Suppose all tendons did not slack with large enough pretension, the change of length of these two tendons should be the same with opposite sign. Therefore:

$$
\delta_{A B}+\delta_{C D}=0
$$

Substitute (15) and (16) into (17), we get the relation between input tension and pretension:

$$
T_{1} v^{-}-T_{3} v^{+}=2 T_{0} L
$$

Let $l_{\text {in }}$ and $l_{\text {out }}$ be the input and output pulling distance of the tendon respectively. For tendon $A B$, the output pulling distance is equal to the difference of the input pulling distance and elongation, i.e.

$$
l_{\text {out }}=l_{\text {in }}-\delta_{A B}
$$

When the input pulley rotates clockwise, the relation becomes:

$$
l_{\text {out }}=l_{\text {in }}-\delta_{C D}
$$

\subsection{Backlash Identification}

If the pretension is large enough that when the input tension increases, the output side can response immediately, i.e. $L_{1}=L . L$ is the total length of the sheath and $L_{1}$ is the distance from the start of the sheath to any point such that the tension becomes constant. If the tension is not enough, $L_{1}$ would smaller than $L$. This induces a backlash called effective backlash that the input tension is not enough to overcome the friction loss in the sheath, which can be described by the following equations:

$$
T(x)= \begin{cases}T_{i n} e^{-\zeta \theta \operatorname{sgn}(\dot{x})} & \text { if } x<L_{1} \\ T_{0} & \text { if } x \geq L_{1}\end{cases}
$$

and

$$
L_{1}=\min \left\{x \in T(x)=T_{0}\right\}
$$

Since tendon $A B$ is at the pulling side and tendon $C D$ is at the releasing side. The net tension input and output can be written in these forms:

$$
\begin{gathered}
T_{\text {in }}=T_{1}-T_{3} \\
T_{\text {out }}=T_{2}-T_{4}
\end{gathered}
$$

By substituting (11) and (12) into (24):

$$
T_{\text {out }}=T_{1} e^{-\zeta \theta}-T_{3} e^{\zeta \theta}
$$

For minimum pretension, there is no tension output. Therefore (27) becomes:

$$
T_{1} e^{-\zeta \theta}-T_{3} e^{\zeta \theta}=0
$$

Expanding (18) and substitutes (25) and (26), we get:

$$
\begin{aligned}
& T_{1} \frac{r}{\zeta}\left(1-e^{-\zeta \theta}\right)-T_{3} \frac{r}{\zeta}\left(1-e^{\zeta \theta}\right)=2 T_{0} L \\
& \frac{r}{\zeta}\left(T_{1}-T_{3}\right)-\left(T_{1} e^{-\zeta \theta}-T_{3} e^{\zeta \theta}\right)=2 T_{0} L
\end{aligned}
$$




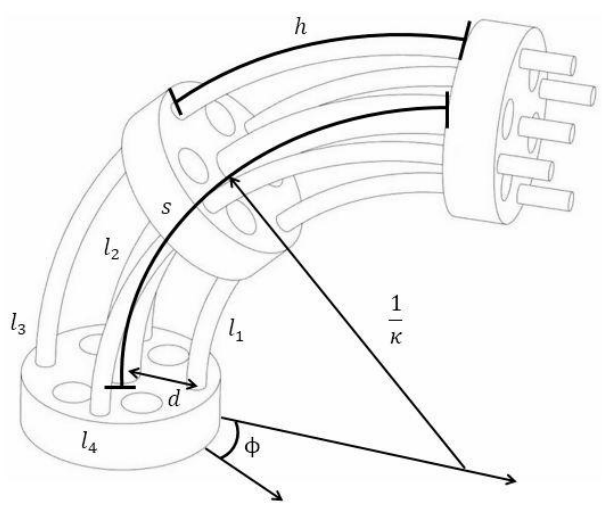

Fig. 4 Segment of continuum manipulator

$$
T_{\text {in }}=2 T_{0} L \frac{\zeta}{r}
$$

If the input tension is smaller than $2 T_{0} L \frac{\zeta}{r}$, there is no tension output. The effective backlash is defined as:

$$
\delta_{0}=\frac{2 T_{0} L}{E}\left(1-e^{-\zeta \theta}\right)
$$

Combining with (15), the overall elongation of the tendon-sheath system becomes:

$$
\delta_{t s}=\left\{\begin{array}{lr}
\delta_{A B}+\delta_{0} & \text { clockwise } \\
\delta_{A B}-\delta_{0} \text { anti }- \text { clockwise }
\end{array}\right.
$$

Suppose $q_{d}$ is the desired trajectory, then the modified trajectory $q_{m}$ can be written as:

$$
q_{m}=q_{d}+\delta_{t s}
$$

\subsection{Force Analysis of Continuum Manipulator}

The kinematic of a 4 wires driven continuum manipulator, as shown in figure 4, is formulated in [25]:

$$
\left[\begin{array}{l}
l_{1} \\
l_{2} \\
l_{3} \\
l_{4}
\end{array}\right]=\left[\begin{array}{l}
s(1-\kappa d \sin \phi) \\
s(1+\kappa d \cos \phi) \\
s(1+\kappa d \sin \phi) \\
s(1-\kappa d \cos \phi)
\end{array}\right]
$$

where

$l_{i}$ is the length of $i$ th actuation tendon,

$s$ is the length of the backbone,

$\kappa$ is the curvature of the continuum manipulator,

$d$ is the distance between actuation tendon and backbone, and

$\phi$ is the rotation angle of the continuum manipulator.

From the study in [29], the following nondimensional parameters are defined as:

$$
\begin{gathered}
h=d / \ell \\
T=F S^{2} / E I
\end{gathered}
$$

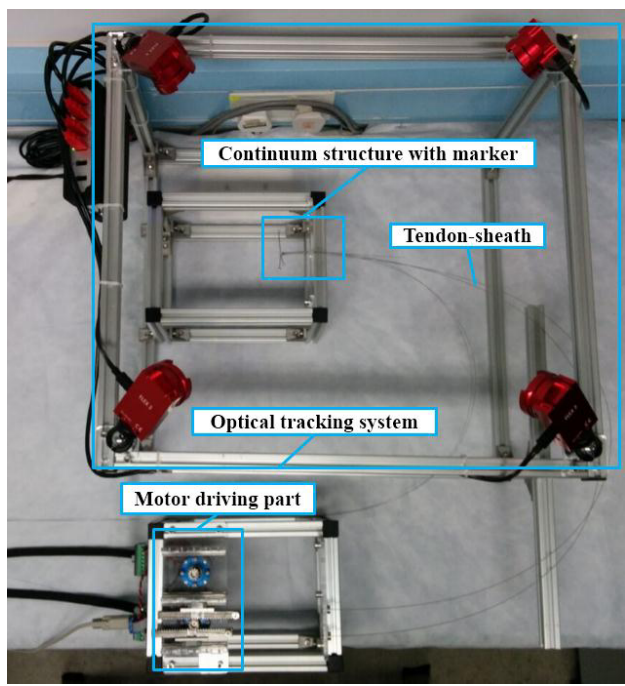

Fig. 5 Experimental setup

where

$h$ is the non-dimensional representation of the geometry of continuum manipulator,

$\ell$ is the distance between each disk,

$T$ is the non-dimensional pulling force,

$F$ is the pulling force, and

$E I$ is the bending stiffness of the backbone.

From the result of this study, the relation between curvature of the manipulator $\kappa$ and pulling force:

$$
\begin{gathered}
\kappa=h T \\
F=\kappa \frac{E I \ell}{d s^{2}}
\end{gathered}
$$

This shows that the pulling force is a function of curvature. The elongation of tendon due to this pulling force can be calculated as:

$$
\delta_{c}=F / E
$$

Combine the result in (31), we get the overall elongation of the system and the input modified trajectory becomes:

$$
q_{m}=q_{d}+\delta_{t s}+\delta_{c}
$$

\section{Model Validation}

\subsection{Experimental Setup}

Figure 5 shows the experimental setup. A $5.6 \mathrm{~mm}$ diameter and $30 \mathrm{~mm}$ long continuum manipulator with disk spacing $5 \mathrm{~mm}$ was used in this experiment. The tendon and the sheath formed a $0.6 \mathrm{~m}$ diameter semicircle lying on supporter. The total length of the sheath was $1 \mathrm{~m}$. An infrared reflective 3-points marker was attached at the tip of the continuum manipulator. An optical tracking system (Flex 3, OptiTrack, NaturalPoint, Inc. OR, USA) was used to track and record the position and the orientation of the marker. Both ends of the tendon 


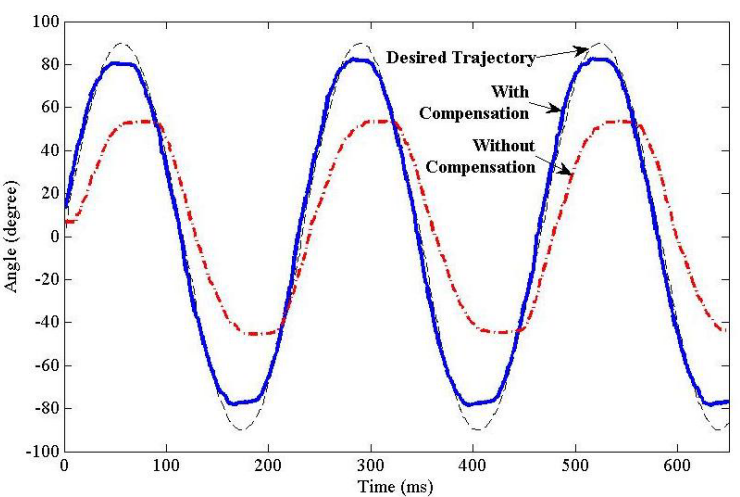

Fig. 6 Experiment result

were attached to the motor driving unit (Faulhaber 3863024C, 1:14 gearbox).

The continuum manipulator was commanded to move between -90 degree to 90 degree on the horizontal plane. The input signal frequency was set at $0.4 \mathrm{~Hz}$. The desired trajectory, trajectory without compensation and trajectory with compensation was plotted in figure 6 .

\subsection{Experiment Result}

The result shows that the continuum manipulator almost followed the desired trajectory. Average error after compensation is less than 5\%. Comparing the trajectory before and after compensation, backlash and elongation are overcome. Delay of action transmission and inaccurate position control are compensated by our feedforward controller. The maximum error occurs at extreme point where the average error is $8 \%$. This error may due to uneven spacing between disks, twisting of the manipulator and compression of the sheath. These uncertainties can be eliminated by better production process and material.

\subsection{Frequency Response of the System}

Since this tendon-sheath actuation system has nonlinear behaviour, therefore the actuation frequency would vary the accuracy. A sinusoidal input from 0.05 to $2 \mathrm{~Hz}$ was used to repeat the above experiment.

The Bode plot of the experiment is shown in figure 7. The result shows that the error magnitude starts to increase from $0.8 \mathrm{~Hz}$ which means $0.8 \mathrm{~Hz}$ is the maximum frequency that can guarantee the above control accuracy, while this bandwidth is enough for surgeon to complete surgical tasks. Control accuracy decreases with frequency can be explained by the respond time of the motor. The accuracy does not change too much at low frequency while the accuracy drops significantly when the frequency increases. It is because the motor cannot follow the input trajectory when the frequency increases.

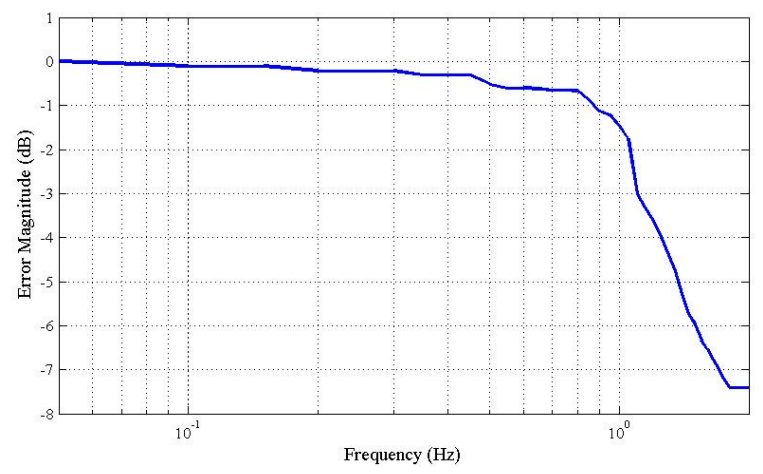

Fig. 7 Frequency response of the system

\section{Conclusion}

This paper presented a model based feedforward motion controller for tendon-sheath driven continuum manipulator. The results show that our proposed model reduces the position error less than $5 \%$. The frequency bandwidth is about $0.8 \mathrm{~Hz}$ which is enough to complete surgical tasks.

The next stage of this research is going to put this model into application. This model will be applied to our endoscopic surgical robot to complete surgical task such as ESD. The completion time and accuracy of the surgery before and after applying this model can be compared to evaluate the effectiveness.

\section{Acknowledgment}

This research is supported by ITF project from Innovation and Technology Commission, HKSAR (Ref. No. ITS/252/13FP).

\section{References}

1. Kaneko, Makoto, Tadashi Yamashita, and Kazuo Tanie. "Basic considerations on transmission characteristics for tendon drive robots." Advanced Robotics, 1991.'Robots in Unstructured Environments', 91 ICAR., Fifth International Conference on. IEEE, 1991.

2. Kaneko, Makoto, et al. "A new consideration on tendontension control system of robot hands." Robotics and Automation, 1991. Proceedings., 1991 IEEE International Conference on. IEEE, 1991.

3. Palli, Gianluca, and Claudio Melchiorri. "Model and control of tendon-sheath transmission systems." Robotics and Automation, 2006. ICRA 2006. Proceedings 2006 IEEE International Conference on. IEEE, 2006.

4. Palli, Gianluca, Gianni Borghesan, and Claudio Melchiorri. "Tendon-based transmission systems for robotic devices: Models and control algorithms."Robotics and Automation, 2009. ICRA'09. IEEE International Conference on. IEEE, 2009.

5. Kesner, Samuel B., and Robert D. Howe. "Design and control of motion compensation cardiac catheters." Robotics and Automation (ICRA), 2010 IEEE International Conference on. IEEE, 2010. 
6. Kesner, Samuel B., and Robert D. Howe. "Position control of motion compensation cardiac catheters." Robotics, IEEE Transactions on 27.6 (2011): 1045-1055.

7. Hyodo, Shoyo, Yujiro Soeda, and Kouhei Ohnishi. "Verification of flexible actuator from position and force transfer characteristic and its application to bilateral teleoperation system." Industrial Electronics, IEEE Transactions on56.1 (2009): 36-42.

8. Suzuki, Yusuke, et al. "Development and verification of tendon-driven rotary actuator for haptics with flexible actuators and a PE line." Advanced Motion Control, 2010 11th IEEE International Workshop on. IEEE, 2010.

9. Low, Soon Chiang, et al. "Master-slave robotic system for therapeutic gastrointestinal endoscopic procedures." Engineering in Medicine and Biology Society, 2006. EMBS'06. 28th Annual International Conference of the IEEE. IEEE, 2006.

10. Phee, Soo Jay, et al. "Master and slave transluminal endoscopic robot (MASTER) for natural orifice transluminal endoscopic surgery (NOTES)."Engineering in Medicine and Biology Society, 2009. EMBC 2009. Annual International Conference of the IEEE. IEEE, 2009.

11. Do, T. N., et al. "Hysteresis modeling and position control of tendon-sheath mechanism in flexible endoscopic systems." Mechatronics 24.1 (2014): 12-22.

12. Wang, Zheng, Zhenglong Sun, and Soo Jay Phee. "Modeling tendon-sheath mechanism with flexible configurations for robot control." Robotica 31.07 (2013): 1131-1142.

13. Sun, Zhenglong, Zheng Wang, and Soo Jay Phee. "Elongation Modeling and Compensation for the Flexible Tendon--Sheath System." Mechatronics, IEEE/ASME Transactions on 19.4 (2014): 1243-1250.

14. Do, T. N., et al. "An investigation of friction-based tendon sheath model appropriate for control purposes." Mechanical Systems and Signal Processing42.1 (2014): 97-114.

15. Agrawal, Varun, et al. "Control of cable actuated devices using smooth backlash inverse." Robotics and Automation (ICRA), 2010 IEEE International Conference on. IEEE, 2010.

16. Agrawal, Varun, William J. Peine, and Bin Yao. "Modeling of transmission characteristics across a cableconduit system." Robotics, IEEE Transactions on 26.5 (2010): 914-924.

17. Agrawal, Vishal, William J. Peine, and Bin Yao. "Dual loop control of cable-conduit actuated devices." American Control Conference (ACC), 2012. IEEE, 2012.

18. Phee, Soo J., et al. "Tendon sheath analysis for estimation of distal end force and elongation for sensorless distal end." Robotica 28.07 (2010): 1073-1082.

19. Do, T. N., et al. "Adaptive control of position compensation for cable-conduit mechanisms used in flexible surgical robots." Informatics in Control, Automation and Robotics (ICINCO), 2014 11th International Conference on. Vol. 1. IEEE, 2014.

20. Sun, Zhenglong, Zheng Wang, and Soo Jay Phee. "Modeling and motion compensation of a bidirectional tendon-sheath actuated system for robotic endoscopic surgery." Computer methods and programs in biomedicine 119.2 (2015): 77-87.

21. Lin, Chen, Wang Xingsong, and Tian Fuxiang. "Tendonsheath actuated robots and transmission system." Mechatronics and Automation, 2009. ICMA 2009. International Conference on. IEEE, 2009.

22. Chen, Lin, Xingsong Wang, and W. L. Xu. "Inverse transmission model and compensation control of a singletendon-sheath actuator." Industrial Electronics, IEEE Transactions on 61.3 (2014): 1424-1433.
23. Wu, Qingcong, et al. "Transmission Model and Compensation Control of Double-Tendon-Sheath Actuation System." Industrial Electronics, IEEE Transactions on 62.3 (2015): 1599-1609.

24. Poon, C. C. Y., Yang, H., Lau, K. C., et al. "A Bio-inspired Flexible Robot with Hybrid Actuation Mechanisms for Endoscopic Surgery." The Hamlyn Symposium on Medical Robotics. 2014.

25. Lau, K. C., et al. "Design and development of a task specific robot for endoscopic submucosal dissection of early gastrointestinal cancers." Optomechatronic Technologies (ISOT), 2014 International Symposium on. IEEE, 2014

26. $\mathrm{Xu}, \mathrm{Wenjun}$, et al. "A feed-forward friction compensation motion controller for a tendon-sheath-driven flexible robotic gripper." Robotics and Biomimetics (ROBIO), 2013 IEEE International Conference on. IEEE, 2013.

27. Lau, K. C., et al. "A Surgical Robotics System for Endoscopic Submucosal Dissection." Industrial Informatics, IEEE Transactions on. (Proceeding)

28. Armstrong-Hélouvry, Brian, Pierre Dupont, and Carlos Canudas De Wit. "A survey of models, analysis tools and compensation methods for the control of machines with friction." Automatica 30.7 (1994): 1083-1138.

29. Li, Changquing, and Christopher D. Rahn. "Design of continuous backbone, cable-driven robots." Journal of Mechanical Design 124.2 (2002): 265-271. 